\title{
GERENCIAMENTO DE ENFERMAGEM NO ENFRENTAMENTO DA COVID-19 NOS SERVIÇOS DE HEMODIÁLISE
}

Joseneide Santos Queiroz ${ }^{1}$

Patricia Figueiredo Marques ${ }^{1}$ https://orcid.org/0000-0001-9938-5067

https://orcid.org/0000-0002.0242-5024

Objetivo: Discutir medidas preventivas, de detecção precoce e estabelecimento de barreiras, no escopo gerencial do trabalho do enfermeiro responsável técnico do serviço de hemodiálise crônica, contra à COVID-19. Método: Trata-se de um artigo de reflexão, que aborda o trabalho do enfermeiro gestor para enfrentamento a pandemia causada pelo coronavírus. Resultados: $\mathrm{O}$ enfermeiro gestor do serviço assume as diretrizes relacionadas a equipe de enfermagem, ao manejo clínico dos pacientes e monitoramento do ambiente físico. Organizar esse fluxo ambulatorial para todos envolvidos, é estabelecer uma barreira efetiva para tratamento precoce e prevenção de danos. Conclusão: O aporte teórico da Qualidade em Saúde, no item da Segurança do Paciente e as diretrizes técnicas das Sociedades de Especialistas em Nefrologia oferecem o subsídio teórico para enfrentar a pandemia no âmbito da gestão da enfermagem, mas o alcance dessas ações será efetivado no exercício profissional da enfermagem. Descritores: COVID-19; hemodiálise; segurança do paciente; educação; enfermagem.

\section{NURSING MANAGEMENT IN FACING COVID-19 IN HEMODIALYSIS SERVICES}

Objective: This paper's aim is to discuss measures of detection and prevention, in the scope of a nurse manager technician responsible for the chronic Hemodialysis service, against COVID-19. Method: This paper describes how a nurse manager should exercise their position in order to face the pandemic caused by the coronavirus. Results: The nurse manager that is responsible for this service will be held accountable for the correct following of the guidelines that are related to their team, the handling of patients and monitoring of their work environment. Conclusion: Organizing these outpatient shifts is essential to ensure an effective barrier for early treatment and general damage prevention in this context. The theoretical contribution of Quality in Healthcare in the entry of Patient Safety and the technical guidelines of the Nephrology Specialists' society offer the theoretical background and support to face the pandemic within the scope of nursing management.

Descriptors: COVID-19; hemodialysis; patient safety; education; nursing

\section{GESTIÓN EN ENFERMERİA ENFRENTANDO COVID-19 EN SERVICIOS DE HEMODIALISIS}

Objetivo: Discutir las medidas preventivas, la detección temprana y el establecimiento de barreras, dentro del alcance administrativo del trabajo de la enfermera a cargo del servicio de hemodiálisis crónica, contra COVID-19. Método: este es un artículo reflexivo que aborda el trabajo del gerente de enfermería para enfrentar la pandemia causada por el coronavirus. Resultados: La enfermera administradora de servicios asume las pautas relacionadas con el equipo de enfermería, el manejo clínico de los pacientes y el monitoreo del entorno físico. Organizar este flujo ambulatorio para todos los involucrados es establecer una barrera efectiva para el tratamiento temprano y la prevención de daños. Conclusión: El aporte teórico de Calidad en Salud, en el item de Seguridad del Paciente y las directrices técnicas de las Sociedades de Especialistas en Nefrología ofrecen el apoyo teórico para enfrentar la pandemia en el ámbito del manejo de enfermería, pero el alcance de estas acciones será efectivo en el ejercicio profesional de enfermería.

Descriptores: COVID-19; hemodiálises; seguridad del paciente; educación; enfermería

${ }^{1}$ Curso de Graduação em Enfermagem, Centro de Ciências da Saúde da Universidade Federal do Recôncavo da Bahia, BA Autora correspondente: Joseneide Santos Queiroz E-mail: josy-queiroz@hotmail.com

Recebido: $26 / 4 / 2020$

Aceito: $23 / 5 / 2020$ 


\section{INTRODUÇÃO}

A COVID-19 é uma doença ocasionada pelo novo coronavirus, sem vacina disponivel, altamente contagiosa, com ausência de recursos terapêuticos de comprovada eficácia e com riscos associados a comorbidades presentes no público de pessoas em Hemodiálise ${ }^{(1)}$.

A terapia renal de substituição é representada por três modalidades de tratamento, que são: hemodiálise, diálise peritoneal e transplante renal. Neste grupo estão elencados vários fatores de riscos para agravamento da infecção pela COVID-19, são hipertensos, diabéticos, idosos, com doenças cardiacas prévias e fatores inflamatórios ocasionados pela uremia ${ }^{(1,2)}$.

Todo esse complexo sistema agora necessita enfrentar as sindromes respiratórias agudas. Nesta população, as medidas para identificação precoce e estabelecimento de barreiras efetivas para evitar contágio, serão preponderantes para salvar mais vidas. O objetivo deste artigo é discutir medidas preventivas, de detecção precoce e estabelecimento de barreiras, no escopo gerencial do trabalho do enfermeiro responsável técnico do serviço de hemodiálise crônica contra à COVID-19. Metodologicamente caracteriza-se como artigo de reflexão elaborado a partir da experiência profissional de uma das autoras e reflexões advindas de recomendações elaboradas pelas sociedades internacionais e nacional de nefrologia sobre o trabalho do enfermeiro gestor para enfrentamento a pandemia causada pelo coronavírus.

\section{A SALA DE HEMODIÁLISE E à COVID-19}

As salas de hemodiálise aglomeram pessoas, pois elas chegam ao serviço com acompanhantes quando não possuem autonomia para o seu deslocamento. Fazem o procedimento na frequência de três vezes por semana, em turnos fixos, que podem ser durante a manhã, tarde ou noite, em dias alternados, durante quatro a cinco horas por sessão. Os leitos estão dispostos de modo que facilita a visualização de todo o ambiente, por não existir barreiras físicas entre um e outro. Esse layout contribui também para distribuição e eliminação da água tratada para diálise, sem a qual não se pode realizar o procedimento $^{(1)}$.

Esta breve descrição condiz com o funcionamento normatizado e cumpre com as exigências padronizadas pela Divisão de Vigilância Sanitária para funcionamento dos serviços de diálise, ao menos até a chegada do novo coronavírus. Todo este complexo sistema passa pela gestão de um enfermeiro(a) responsável técnico(a), que possui título de Especialista em Nefrologia e assume as diretrizes relacionadas à equipe de enfermagem, ao manejo clínico dos pacientes e monitoramento do ambiente físico.

Um indivíduo portador assintomático da COVID-19 facilmente pode infectar várias pessoas durante a sessão de hemodiálise, respirando em ambiente confinado. Dentro da sala de procedimento ficam os profissionais e os pacientes, na maioria das vezes em uso de ar condicionado para manter temperatura de conforto. Esta descrição permite identificar a necessidade de orientação e determinação de estratégias para enfrentamento da COVID-19 para três grupos de pessoas envolvidas nessa dinâmica de funcionamento: pacientes, seus acompanhantes e profissionais.

Estabelecido os três grupos de interesse, serão abordados os aspectos da Segurança do Paciente que serão gerenciados pela Enfermeiro(a) Responsável Técnico(a) pelo serviço.

\section{SEGURANÇA DO PACIENTE E MITIGAÇÃO DE RISCOS}

A Segurança do Paciente de acordo com a Organização Mundial da Saúde caracteriza-se pela redução do risco de danos indevidos associados ao cuidado em saúde até um mínimo aceitável(3). O serviço de nefrologia precisará estabelecer condutas pensadas para a "Instituição" e para o conjunto de pessoas que frequentam suas dependências.

Avaliando a contaminação pela COVID-19, a propagação se dá por aerossóis e fixação em superfícies, que serão transportadas pelas mãos e podem alcançar a mucosa oral, nasal e ocular, viabilizando o adoecimento(2). Diferente da maior parte das doenças de transmissão sanguínea, para as quais os cuidados já estão estabelecidos na hemodiálise, a transmissão respiratória da COVID-19 desafia a logística de funcionamento do serviço e o problema precisa ser tratado com vigilância a saúde dos grupos de interesse.

O primeiro momento é de estabelecer regras gerais para o monitoramento de sinais e sintomas e classificação de risco. Várias recomendações e protocolos têm sido apresentados, orientando e direcionando as ações específicas para os pacientes em terapia renal substitutiva, como a proposta pela European Dialysis (EUDIAL) Working Group of ERA-EDTA(1), as orientações da Sociedade Brasileira de Nefrologia ${ }^{(2)}$ e o Protocolo de manejo clínico da COVID-19 na Atenção Primária à Saúde(4). Esse referencial subsidia essa discussão sobre o estabelecimento de diretrizes de funcionamento do serviço de hemodiálise crônica no combate a pandemia.

O essencial é entender que estes documentos fonte estabelecem fluxos para vigilância em saúde, que são adaptados para o monitoramento de todo grupo de pessoas que estão vinculados ao serviço de nefrologia. Os protocolos que venham a ser desenvolvidos pelos serviços devem incluir os intervalos sem diálise para pacientes, familiar/ acompanhante, funcionários do serviço. O surgimento de sintomas levará para avaliação médica e indicações de isolamento ou internação. Organizar esse fluxo é estabelecer uma barreira efetiva para tratamento precoce e prevenção de danos.

Diante desse contexto pandêmico, todos os serviços de hemodiálise independentemente da natureza jurídica ser pública, privada ou filantrópica precisam rever o fluxo de atendimento, isolamento e/ou internamento quando 
identificados casos positivos para à COVID-19. O serviço público está redimensionando seu atendimento e é necessário se antecipar, discutir e redirecionar este encaminhamento para que os pacientes e familiares possam buscar ou serem regulados para atendimento adequado.

Quanto aos profissionais de saúde, eles estão expostos ao contágio e podem se tornar portadores assintomáticos, piorando a situação de exposição dos pacientes, considerando ser este o pior cenário devido as comorbidades. É necessário estabelecer um fluxo novo, específico para este momento da pandemia, no qual os serviços de Nefrologia ao propor as ações de barreira reconheçam o "bioma" constituído por pacientes, familiar/ acompanhante, funcionários.

Os pacientes necessitam permanecer em isolamento social e são necessários vários cuidados com o ambiente domiciliar. O cenário é de muita informação, mas este grupo de pessoas dependentes de hemodiálise estão adaptados a sobreviver com muitas restrições, inseridas no contexto do procedimento dialítico, e recebem orientações de Educação para Saúde no serviço ${ }^{(1,2,4)}$.

O segundo item, também abrangente, é a avaliação de todos os procedimentos de higienização, sendo incluídos novos itens na rotina dos grupos de interesse. Cabem o uso das técnicas de Educação para Saúde ativas e reflexivas direcionadas para importância da instrução para o autocuidado e na realização dos procedimentos. Será necessário a modificação de hábitos na chegada para a sessão de hemodiálise, assumindo novas rotinas como o uso de máscaras pelos pacientes, cuidados no deslocamento no transporte público urbano ou de transporte fora do domicilio, no momento do lanche, entre outros procedimentos operacionais de higienização no próprio serviço de diálise $\mathrm{e}^{(1,2,4)}$

A Educação Permanente será necessária para estabelecer treinamentos com a especificidade da área de atuação profissional e o uso das Metas Internacionais para Segurança do Paciente, com destaque para as metas de Higiene das Mãos e da Comunicação Efetiva. Estas metas serão mais abordadas pela necessidade de estabelecer protocolos e difusão do conhecimento com a padronização de Equipamentos de Proteção Individual e Coletiva (EPI e EPC), com foco no enfrentamento da COVID-19(5). Este é um item sensivel, uma vez que existe adoecimento relacionado a erros ao se paramentar $e$ desparamentar durante a jornada de trabalho(1). O serviço deve dispor os insumos, a técnica precisa ser treinada, e a equipe precisa ser motivada a cuidar-se para manter-se saudável.

Ainda na questão do adoecimento profissional, o serviço de terapia renal de substituição é uma área especializada e é difícil encontrar profissionais habilitados para contratação e atuação imediata. Este deve ser um item a se pensar, estabelecer metas para admissão e aperfeiçoamento de pessoal para manter a capacidade de atendimento.

Amodificação no fluxo promovea inserçãovariada na compra de EPI e EPC que são dispendiosos, geralmente provocam um primeiro pensamento sobre o aumento de custos. No entanto, a pandemia provocada pela COVID-19 tem demonstrado que o momento requer enxergar como investimento, os melhores resultados são em vidas salvas. Por certo, há necessidade de discussão com os setores responsáveis pelo orçamento do Sistema Único de Saúde e Convênios de Saúde para a modificação nos valores das sessões de hemodiálise, e fazer a compilação de dados objetivos para entender o plano de enfrentamento da pandemia, podem fomentar o entendimento dos resultados alcançados em custo-efetividade.

\section{CONCLUSÕES}

O desafio para o enfermeiro(a) Responsável Técnico(a) do serviço de Hemodiálise e gestores hospitalares que prestam esse cuidado se impõe num cenário de incertezas. Enquanto avançam as pesquisas, o melhor referencial para assistência segura é o embasamento em evidências. O aporte teórico da Qualidade em Saúde, no item da Segurança do Paciente e as diretrizes técnicas das Sociedades de Especialistas em Nefrologia e Ministério da Saúde oferecem o subsídio teórico para enfrentar a pandemia da COVID-19, mas o alcance dessas ações será efetivado no exercício profissional da enfermagem.

CONTRIBUIÇÕES DOS AUTORES: Joseneide Santos Queiroz contribui para esse artigo com: a concepção e/ou desenho do estudo; coleta, análise e interpretação dos dados; redação e/ ou revisão crítica do manuscrito, aprovação da versão final a ser publicada; Patrícia Figueiredo Marques contribui para esse artigo com: a concepção e/ou desenho do estudo; coleta, análise e interpretação dos dados; aprovação da versão final a ser publicada.

\section{REFERÊNCIAS}

1. Basile C, Combe C, Pizzarelli F, Covic A. Davenport A, Kanbay M, et al. Recommendations for the prevention, mitigation and containment of the emerging SARS-CoV-2 (COVID-19) pandemic in haemodialysis centres.Nephrol Dial Transplant. 2020:2:1-4. Disponivel em https://www.ncbi.nlm.nih.gov/pubmed/32196116; acesso em 20 de abril de 2020 .

2. Sociedade Brasileira de Nefrologia. Recomendações da Sociedade Brasileira de $\mathrm{Ne}$ frologia às Unidades de Diálise em relação a Epidemia do novo Coronavirus (COVID-19) [Internet]. 2020. Available from: http://sbn.org.br/ 20 de abril de 2020.

3. Negrão SMC, Conceição MN, Mendes MJF, Araújo JS, Pimentel IMS, Santana ME. Avaliação da prática de enfermagem na segurança do paciente oncológico. Enferm em

Foco. 2019:10(4):136-41. Disponivel em http://revista.cofen.gov.br/index.php/enfermagem/article/view/2129. Acesso em 20 de março de 2020.

4. Ministério da Saúde (BR). Secretaria de Atenção Primária à Saúde. Protocolo de manejo clinico da COVID-19 na Atenção Primária à Saúde. 2020. Disponivel em https://www.saude.gov.br/images/pdf/2020/marco/20/20200318-ProtocoloManejo-ver002.pdf. Acesso em 20 de abril de 2020.

5. La Regina M. Tanzini M, Venneri F, Toccafondi G, Fineschi V, Lachman P, et al. Patient safety recommendations for COVID-19 epidemic outbreak. J Glob Clin Eng 2020:(3):3-30. Disponivel em https://www.globalce.org/index.php/GlobalCE/article/view/94, Acesso em 20 de abril de 2020 . 